\title{
Aufs Lebensende vorbereiten
}

\author{
Nur mit einem interdisziplinären Ansatz kann Menschen am Lebensende würdig geholfen \\ werden, so das Fazit des Nationalen Palliative Care Kongresses. Essentiell dafür ist, dass man die \\ Kompetenzen anderer Fachgebiete anerkennt - das gilt insbesondere für Ärzte.
}

\section{Felicitas Witte}

Dr. med., Medizinjournalistin

«Noch nie wurde einsamer gestorben als heute.» Als Daniel Infanger, Pfarrer aus Bern, diesen Satz sagt, kommen Bilder in einem hoch: von Menschen, die alleine zu Hause in einem Bett liegen, Bilder von todkranken Patienten, für die Ärzte und Pflegepersonal keine Zeit haben, Bilder von hilflosen Angehörigen, die nicht wissen, an wen sie sich mit ihrer Trauer wenden können. "Wir haben zunehmend die Tendenz, das Sterben vom Leben fernzuhalten", sagte Infanger. Das lässt sich nur gemeinsam ändern, so das Fazit der Experten auf dem Nationalen Palliative Care Kongress, der kürzlich in Biel stattfand*. «Krankheiten werden immer komplexer, gleichzeitig ermöglichen Fortschritte neue Therapien", sagte Steffen Eychmüller, Vizepräsident von palliative ch, der Schweizerischen Gesellschaft für Palliative Medizin, Pflege und Begleitung. «Hinzu kommen die immer steigenden Kosten im Gesundheitssystem. Um diese Herausforderungen bewältigen zu können, müssen wir bereits in der Aus- und Weiterbildung Schnittstellen zwischen den Ausbildungsgängen verschiedener Gesundheits- und Sozialberufe anbieten. Nur so können wir in einem frühen Stadium die Fachkompetenzen vermitteln, um Patienten einfühlsam und kompetent am Lebensende und beim Sterben begleiten zu können.»

\section{Das Studium bereitet auf den Tod nicht vor}

Wann haben Sie zum ersten Mal einen Patienten beim Sterben begleitet? Oder haben Sie das noch nie? Viele Ärzte fühlen sich auf diese Situation nicht gut vorbereitet. Wie können sie auch, wenn sie während ihrer Ausbildung nie damit zu tun haben. «Wir lernen viel und gleichzeitig nichts über das Sterben", sagte Manuel Schaub, Medizinstudent aus Bern, der mit seinem ernsten und gleichzeitig mit viel Humor gespickten Vortrag das Publikum fesselte. «Wir sind nach unserem Studium in der Lage zu erklären, was passiert, wenn eine Zelle im Körper aufhört zu funktionieren. Wir wissen alles Mögliche über Gerätschaf- ten, die den Tod verhindern sollen und oft auch können. Wir kennen von vielen Krankheiten die Wahrscheinlichkeit, daran zu sterben und wir wissen, wie man diese Wahrscheinlichkeit verändern kann. Aber wie das mit dem Sterben geht, wissen wir nicht.» Zwar sei es ein guter Ansatz, dass im fünften Studienjahr einige Vorlesungen über Palliativmedizin angeboten würden, die auch gut besucht seien. «Trotzdem hinterlässt die Tatsache, dass wir in unserem Studium mehr Zeit damit verbringen, das Hebelgesetz zu lernen und Widerstände in elektrischen Schaltkreisen zu berechnen, als sich mit dem Sterben zu beschäftigen, bei mir ein flaues Gefühl», sagte Schaub. Viele Studierende hätten das Bedürfnis, mehr über Palliative Care zu wissen. Die Vereinigung der Schwei-

Man könne nur interprofessionell arbeiten, sagte Eychmüller, wenn man die Kompetenzen der anderen kenne und wertschätze.

zer Medizinstudierenden hat deshalb ein Projekt gestartet mit Namen «doctors and death». In einem eintägigen Workshop diskutieren die Teilnehmer mit Palliativmedizinern, Ethikern, Seelsorgern und Psychologen und besuchen eine Palliativstation oder ein Hospiz. «Der Kurs ist beliebt und jeweils schnell ausgebucht», erzählte Schaub. Wie wichtig Interdisziplinarität in der Palliativmedizin ist, merkte er als junger Student in Wien. Oberschwester Fräulein Gerti, "so streng wie ihr Name und ihre Berufsbezeichnung», war die erste, die ihm gezeigt habe, wie er mit einer Situation eines sterbenden Patienten umgehen könne. "Sie hat mir während der Nachtschicht als Einzige angesehen, dass ich mit dem Tod des Patienten überfordert bin», erzählte Schaub. "Oberschwester Fräulein Gerti hat dann das Fenster geöffnet, um die Seele des Patienten rauszulassen und dazu in breitem Wienerisch gesagt «Dommit hams olls gmocht, was kenn'n und nu is gut.ı" Selbst als bekennender Atheist habe ihm die frische Luft gut getan. «Es ist so wichtig, dass wir angehenden Ärzte einen persönlichen Weg im Umgang mit solchen Situationen finden und lernen, wie der aussehen könnte. Das habe 


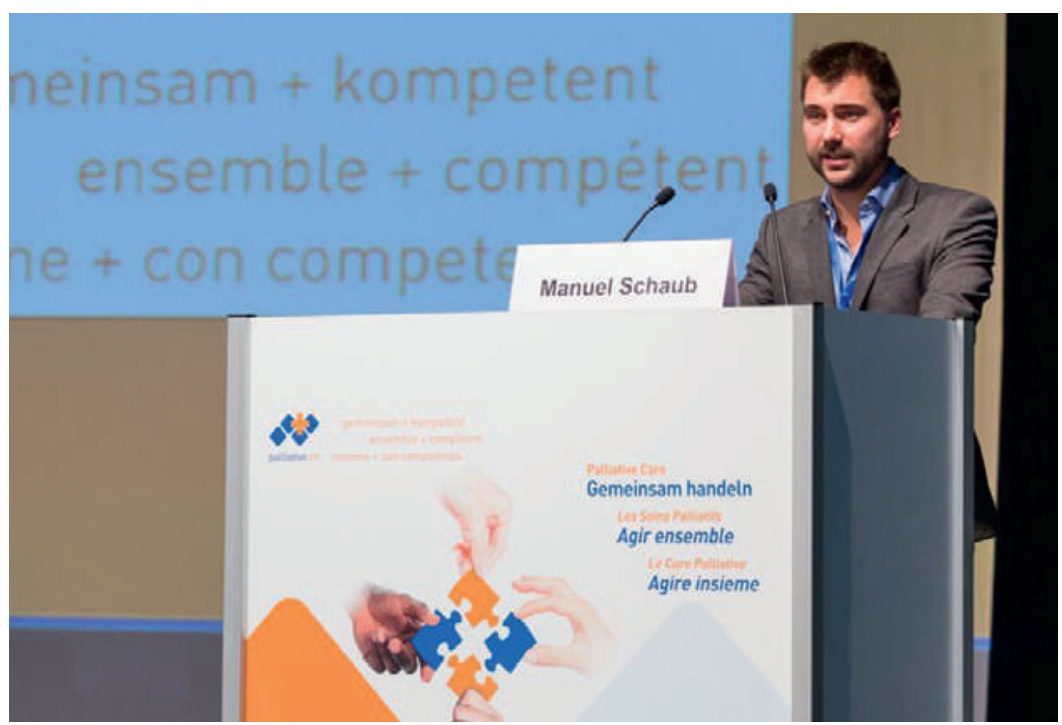

Medizinstudent Manuel Schaub: «Wir verbringen im Studium mehr Zeit damit, das Hebelgesetz zu lernen, als uns mit dem Sterben zu beschäftigen.»

ich nicht von einer Ärztin oder einem Arzt gelernt, sondern von dieser Schwester. So eine wie Fräulein Gerti müsste jeder Student einmal treffen.»

\section{Eine Pflegefachfrau kann manches besser}

Wie es gelingen kann, Palliative Care interprofessionell zu gestalten, war einer der Schwerpunkte auf dem Kongress. "Ohne einen interdisziplinären Ansatz geht es nicht», sagte Palliativmediziner Eychmüller. Die Bereiche, in denen Herausforderungen aufträten, seien sehr vielfältig. «Deshalb braucht es verschiedene Kompetenzen, die eine Berufsgruppe unmöglich alle abdecken kann: von der Symptombehandlung über Entscheidungsfindung, der Planung und Umsetzung eines Netzwerkes für zu Hause oder für die Pflege in einem Hospiz inklusive Notfallplanung bis zum Support der Angehörigen, die massiv belastet sind.» Voraussetzung sei, dass sich die einzelnen Berufsgruppen als "Teamplayers» sähen. «Mediziner haben es hier besonders schwer», erzählte Eychmüller, «weil sie traditionell als 〈Leader〉 trainiert werden, und so haben manche Mühe, die Kompetenz anderer anzuerkennen, etwa der Pflegenden.» Das merkt immer wieder auch Roswitha Koch, Leiterin Abteilung Pflegeentwicklung beim Schweizer Berufsverband der Pflegefachfrauen und Pflegefachmänner SBK. "Es gibt einfach Elemente, die ich als Pflegefachfrau besser verstehe als ein Arzt. Ich wünsche mir, dass Mediziner unsere Autonomie im Bereich Pflege anerkennen, wie wir das anders herum schliesslich auch tun.» Palliative Care schliesst medizinische Behandlungen, Pflege sowie psychologische, soziale und spirituelle Unterstützung mit ein. "Mit partnerschaftlichen Ansätzen, etwa in dem sich die Beteiligten an einem «runden Tisch zusammensetzen, können wir den Patienten am besten behandeln, betreuen und begleiten", sagte Koch. Ein ideales Modell der Interprofessionalität, so stellt sich die Pflegefachfrau vor, orientiert sich am Patienten und strebt die grösstmögliche Qualität an. «Die Fachkompetenz der beteiligten Berufsgruppen muss wegweisend sein, nicht traditionelle Hierarchien oder Standesinteressen.» Im Zuge der demographischen Entwicklungen mit mehr älteren, chronisch kranken Menschen sei eine neue Verteilung der Aufgaben zwischen den Ärzten und nichtärztlichen Mitarbeitern absolut zentral.

«Palliative Care kümmert sich um Menschen in ihrer letzten Lebensphase, die an unheilbaren, lebensbedrohlichen oder chronisch fortschreitenden Krankheiten leiden", sagte Christoph Cina, Vertreter Hausärzte Schweiz und Allgemeinarzt im MediZentrum in Messen. "Damit diese Menschen eine ihrer Situation angepasste optimale Lebensqualität erleben können, braucht es die Unterstützung durch verschiedene Berufsgruppen. Das können wir nur erreichen, wenn verschiedene Fachleute zusammenarbeiten.»

\section{Ein aktiver Lernprozess}

Die Interprofessionelle Zusammenarbeit sei ein aktiver Lernprozess, sagte Cina, der bereits in der Ausbildung durch gemeinsame Module umgesetzt werden müsse. Der Allgemeinarzt erinnert sich an eine Frau mit einer progredienten neurodegenerativen Erkrankung, die sehr unter Sprach-, Schluck- und Gehstörungen litt. «Unter Einbezug verschiedener Fachpersonen wie Physiotherapeuten, Logopäden und Ergotherapeuten konnten wir die Lebensqualität der Frau enorm verbessern", erzählte Cina. "Sie konnte wieder besser kommunizieren, konnte sich besser ernähren und war mobiler durch Anpassungen und Hilfsmittel zu Hause.» Die Strategie müssten die Fachpersonen am «runden Tisch» absprechen, zusammen mit Patient und Angehörigen. "Wir haben uns natürlich nach den Wünschen und Bedürfnissen des Patienten zu richten. Und immer wieder müssen wir unsere Strategie überprüfen, ob der Patient auch davon profitiert und ob sie ihm gut tut.»

Man könne nur interprofessionell arbeiten, sagte Eychmüller, wenn man die Kompetenzen der anderen kenne und wertschätze. "Ausserdem darf man sich selbst nicht als Höhepunkt der akademischen Schöpfung sehen. Man muss Freude an der Selbstrelativierung haben mit der Frage: Worin bin ich gut, 
wo sind andere besser?» Vor allem englischsprachige Länder oder Skandinavien seien in dieser Hinsicht viel weiter. «Dort ist das 〈Standesdünkel〉 der Ärzteschaft geringer, und das Pflegepersonal fühlt sich deutlich wertgeschätzter als bei uns." Man handle dort pragmatisch und problemlösend, «und mit einem Lächeln über sich selbst». Doch auch hierzulande gebe es positive Beispiele einer interprofessionellen Zusammenarbeit, erzählte Eychmüller, von kleinen Teams in der Grundversorgung bestehend aus Hausarzt und Spitex-Mitarbeitenden bis hin zu hochspezialisierten Teams, etwa aus Onkologen, Hausarzt, Pflegepersonal, Hospiz und Seelsorgern. Auch Cina sieht positive Trends. «Die von Bund und Kantonen lancierte Nationale Strategie Palliative Care hat ein erfreuliches Umdenken bewirkt. Palliative Care wurde zu einem Vorreiter eines gemeinsamen Handelns, was auch schon in die Lernzielkataloge des Medizinstudiums eingeflossen ist.» So gäbe es bereits in der Schweiz einen Lehrstuhl für Palliative Care in Lausanne und "vielleicht ist bald die Zeit reif für einen interprofessionellen Lehrstuhl.» Und einen eigenen Facharzt in Palliativmedizin? «Die Implementierung eines Facharztes Palliative Care würde Jahre in Anspruch nehmen und hätte zurzeit wohl kaum eine Chance», sagte jedoch Cina. Die Fachgruppe Ärzte palliative ch arbeitet gerade daran, einen Fähigkeitsausweis für spezialisierte Palliative Care einzuführen. «Dieser hat in der Schweiz die grösste Chance, rasch eingeführt zu werden. Damit wäre ein erster wichtiger Schritt getan.»

\section{Ein Facharzt für Palliativmedizin?}

Auch Steffen Eychmüller glaubt, die Hürden für einen Facharzt in Palliativmedizin seien zurzeit noch $\mathrm{zu}$ hoch. "Palliativmedizin riecht nach <unmedizinisch», und damit auch nach unnötig. Denn Palliativmedizin hält Ärzten immer wieder einen Spiegel vor, dass sie das Sterben als Niederlage der eigenen Leistungsfähigkeit sehen könnten.» In anderen Ländern, die heute einen eigenen Facharzt für Palliativmedizin haben, wie Australien, Kanada, Deutschland, England, Frankreich oder Polen, hätte es vor der Einführung die gleichen Bedenken gegeben. «Eigentlich geht es einerseits um die Wiedereinführung des gesunden Menschenverstandes in die Medizin, und erst in zweiter Linie darum, dass ein Palliativ-Facharzt spezifisches Wissen um Schaden und Nutzen am Lebensende vermitteln kann", sagte der Palliativmediziner. Wegen des "gesunden Menschenverstandes» komme es immer wieder zu Reibereien. «Was wirklich spezifisch ist für Palliativmedizin, da streiten sich die Geister», so Eychmüller, "vor allem in einem ärztlichen Umfeld, in dem neue Strömungen eher als Gefährdung der eigenen Macht- und Verdienstbasis erlebt werden. Wir möchten aber niemandem etwas wegnehmen, sondern mit dem spezifischen Knowhow der Medizin ein Vorgehen für den Umgang mit dem schwersten Thema zurückgeben: Was tun, wenn die ärztliche Heilkunst nichts mehr anbieten kann.» Als Wilfried Schnepp und seine Kollegen 2005 den Lehrstuhl für familienorientierte und gemeindenahe Pflege an der Uni Witten Herdecke gründeten, hätten sie "wie die Jungfrau vor dem Kind gestanden». Inzwischen hat sich durch sein Wirken die Palliativmedizin in Deutschland massgeblich geändert, und Schnepp gilt auch international als Vorbild im Bereich Palliative Care. Wie wichtig Interdisziplinarität ist, erzählte er an einer berührenden Patientengeschichte. An einem Abend im Hospiz habe er einen jungen Mann aufgenommen, der aus einem Akutkrankenhaus kam. «Ein schnell wachsender Tumor im Bauch, die Beinvenen waren massiv gestaut - es war klar, dass er nicht mehr lange leben würde.» Die Pflegerin hat ihm ein Bad angeboten, was der Mann bei Kerzenschein sichtlich genoss. Als die Pflegerin ihn fragte, was er essen möchte, fing er an zu weinen. «Seit zwei Wochen in der Akutklinik, also seitdem er auf den Hospizplatz wartete, hatte keiner mehr mit ihm gesprochen. Für die Ärzte und in der Akutklinik war die Behandlung beendet. Aber der Patient hat gelitten. Das zeigt uns, wie wichtig Palliative Care ist.» Eine gute Betreuung am Lebensende könne nur erreicht werden, wenn man individuell auf die Bedürfnisse eingehe und interprofessionell zusammenarbeite - das sei schon in der Definition der WHO von 2002 ein zentraler Punkt. "Palliative Care meint Interprofessionalität, das geht gar nicht anders!», sagte Schnepp. «Denn ohne das kann man auf die komplexen Bedürfnisse der Patienten gar nicht eingehen.» Dafür brauche es jedoch Politiker, die diese Strategie unterstützen. Als Schnepp über den Tod seiner Mutter erzählte, herrschte betroffenes Schweigen im Publikum. Kurz vor ihrem Tod habe der Arzt im Spital gesagt: "Herzinsuffizienz, dekompensiert, die stirbt morgen.» "Der Kollege hat einfach nur gesehen, dass sein Akutauftrag beendet ist und die Patientin entlassen wird. Damals hat niemand nach Palliative Care gefragt.»

«Es wäre schön, wenn noch mehr Ärzte aus Ihrer «Wagenburg〉 hervorkommen und den anderen Gesundheitsberufen mit Offenheit und Respekt entgegentreten würden», sagt Pflegefachfrau Koch. «Das wäre ein erster Schritt für wahre interprofessionelle Zusammenarbeit.» 